\title{
CT Guided Pudendal Nerve Block
}

\author{
Howard M. Richard III ${ }^{{ }^{*}}$, Richard P. Marvel $^{2}$ \\ ${ }^{1}$ University of Maryland School of Medicine, Baltimore, USA \\ ${ }^{2}$ Center for Pelvic, Pain, Anne Arundel Medical Center, Annapolis, USA \\ Email: *hrichard@umm.edu
}

Received November 26, 2012; revised December 25, 2012; accepted January 2, 2013

\begin{abstract}
Purpose: Retrospective review of CT-guided pudendal nerve blockade for chronic pelvic pain caused by pudendal neuralgia. Materials \& Methods: The study included 23 patients (average age, 40.6 yr; range, 25 - 71 yr) diagnosed with pudendal neuralgia. Diagnostic criteria were: pain in the anatomic distribution of the pudendal nerve, pain worsening with sitting, pain relieved by lying down, and no sensory loss on examination. Alternative etiologies were considered and, if present, patients were excluded from the study. Patients underwent 1 - 4 CT-guided pudendal nerve blocks, using 22-gauge spinal needles placed with intermittent CT guidance. The needles were directed to the expected path of the pudendal nerve, either immediately caudal to the ischial spine or in Adcock's canal. Nerve blocks were performed with 1 cc depot methylprednisolone (40 mg/cc), Bupivacaine 0.25\% (3 cc) and Lidocaine $2 \%$ (2 cc). Procedure times were recorded. Patients were examined for perineal and vulva anesthesia $1 \mathrm{~h}$ after the blocks were performed. Patients' records were reviewed for procedural success and complications. Results: Procedure time was 17.1 min (range, 11 - 26). Twenty-one blocks were performed at the ischial spine, and 2 were performed at the pudendal canal. Twenty-two (95.6\%) of 23 patients reported perineal analgesia after the injection was performed, for a procedural success rate of $96 \%$. One patient developed bilateral leg weakness which resolved in 6. Conclusion: Pudendal nerve block can be performed with CT guidance in a safe and effective manner. Further evaluation of the long-term effectiveness of the procedure with a prospective study is warranted.
\end{abstract}

Keywords: Pain Management; CT Guidance; Neuropathy

\section{Introduction}

Chronic Pelvic pain has a variety of causes. One of these is pudendal neuralgia. Pudendal neuralgia is a compression neuropathy similar to carpal tunnel syndrome [1-3]. This diagnosis is often one of exclusion. Pudendal neuralgia is a pain syndrome comprised of pain in the cutaneous distribution of the pudendal nerve. The pudendal nerve is a predominately sensory nerve arising from s2 4 nerve roots. The nerve has three branches, the inferior rectal, peroneal and dorsal nerve of the penis/clitoris. The first branch innervates the skin around the anus. The second branch has a deep motor portion and two sensory portions, the medial and lateral labial (scrotal) branches. The third branch runs along the skin of the penis/clitoris, innervating the overlying skin. Pudendal neuralgia can present with pain in the distribution of some or all of these branches. We review our initial experience in treating these patients with CT guided pudendal nerve blockade with local anesthetic and corticosteroids. We hope to demonstrate that intervention radiologist can safely perform this procedure.

\footnotetext{
"Corresponding author.
}

\section{Materials and Methods}

IRB approval was sought and obtained for this retrospective study. The study included 23 consecutive patients (age mean 40.6, range 25 - 71) diagnosed with pudendal neuralgia. Diagnostic criteria were: pain in the anatomic distribution of the pudendal nerve, pain worsened with sitting, pain relieved by lying down and no sensory loss on physical examination [1,3]. Alternative etiologies (such as purely coccygeal pain, gluteal or hypogastric pain) were considered, and if present, prospective participants were excluded from the study.

Patients underwent 1 - 4 CT-guided pudendal nerve blocks, using 22-gauge spinal needles (Avid Medical, Toana, VA, USA) placed with intermittent CT guidance. Patients received additional nerve blocks after minimum four weeks' time depending on the response to the initial nerve blockade. In general repeat nerve blocks were not performed unless the patient symptoms warranted additional treatment. The needles were directed to the expected path of the pudendal nerve [4-6], either immediately caudal to the ischial spine (Figure 1) or in Alcock's canal (Figure 2). Initially patients were treated with blockade at the level of initial spine (which is caudal to 


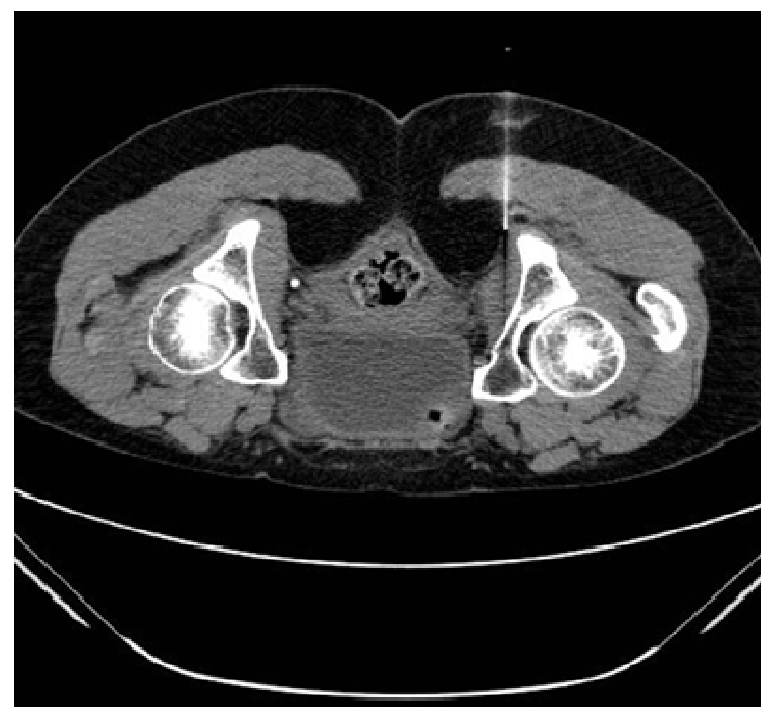

(a)

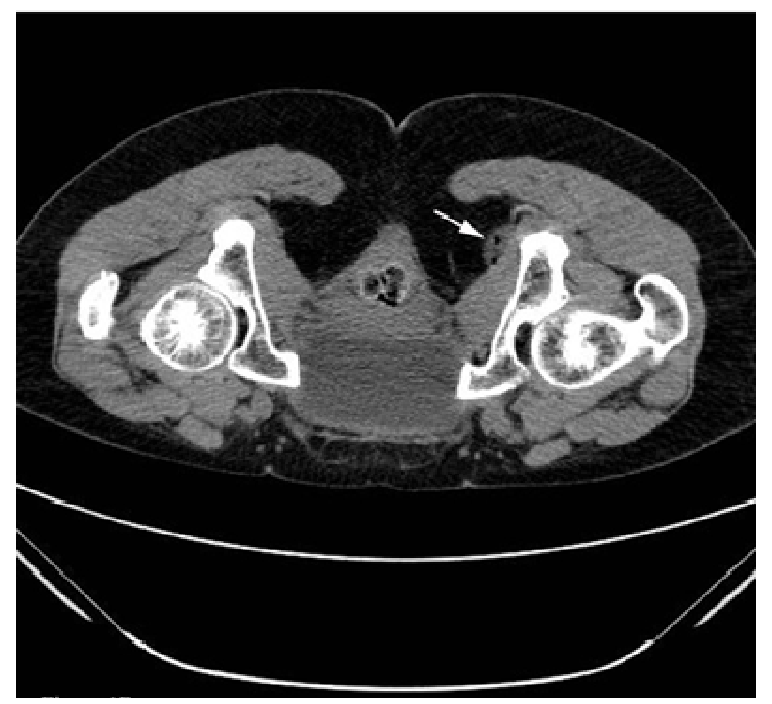

(b)

Figure 1. (a) demonstrates the needle tip immediately caudal to the ischial spine; (b) demonstrates air and fluid (arrow) at the site.

Alcock's canal). Patients who returned with persistent symptoms were treated with the pudendal nerve blockade at Alcock's canal.

Nerve blocks were performed with injection of 1 cc depot methylprednisolone (40 mg/cc) admixed with Bupivacaine $0.25 \%$ (3 cc) and Lidocaine $2 \%$ (2 cc). The CT scanner control software recorded the procedure times automatically. Patients were examined for perineal and vulva anesthesia $1 \mathrm{~h}$ after the blocks were performed as per routine. Patients' records were reviewed for procedural success and complications.

\section{Results}

The average procedure time was 17.1 min (range, 11 -

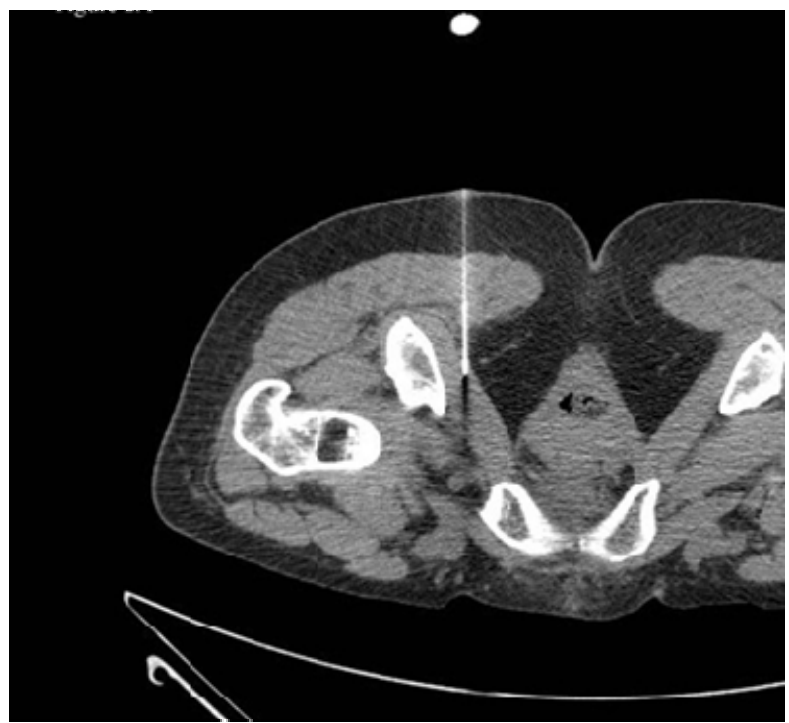

(a)

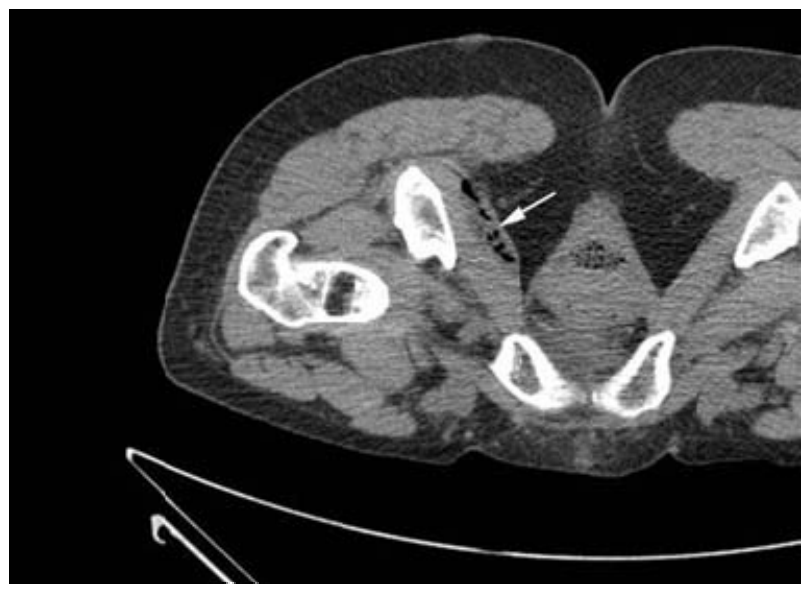

(b)

Figure 2. (a) demonstrates the needle tip in Alcock's (Pudendal) canal; (b) demonstrates air and fluid (arrow) distending the space.

26). Twenty-one blocks were performed at the ischial spine (Figure 2(a)), and 2 were performed at the pudendal canal (Figure 2(b)). Twenty-two (95.6\%) of 23 patients reported perineal analgesia after the injection was performed, for a procedural success rate of $96 \%$. The 1 patient who reported no perineal analgesia was being treated with her fourth injection. Complications were observed in 1 of 23 injections (4.3\%). This patient developed bilateral leg weakness consistent with blockade of the sciatic nerve. In the one patient who had sciatic nerve blockade, the position of the needle tip was slightly cephalad to the ischial spine. The leg weakness resolved in $6 \mathrm{~h}$.

\section{Discussion}

Chronic perianal perineal pain is a debilitating condition. 
This type of neuropathy is typically caused by compression of the pudendal nerve. This compression of the pudendal nerve was first described by Amareno et al. as pudendal canal syndrome [7]. Diagnostic criteria for Pudendal neuralgia has been elucidated by a multidisciplinary working group that met in Nantes France [8]. The five essential criteria are: 1) pain along the anatomical distribution of the pudendal nerve; 2) pain aggravated $b$ sitting; 3) the patient is not awaken at night by the pain; 4) there is no objective sensory loss on clinical examination; and 5) the pain is improved with an anesthetic pudendal nerve block. Neuropathies are typically caused by nerve trunk compression, faulty nutrition, toxins and inflammation [3].

The pudendal nerve is a predominately sensory nerve arising from S2, S3 and S4. It contains some motor nerve fibers which can innervate the external anal and external urethral sphincters. The nerve follows the posterior aspect of the sacrospinous ligament, and then passes anterior to the sacrotuberous ligament. The pudendal nerve is at its closest association with the sciatic nerve between the piriformis and coccygeus muscles just cephalad to the ischial spine [3]. The nerve then passes around the ischial spine before entering the pudendal canal of Alcock. This is a space between the obturator internus fascia on the lateral wall of the ischioanal fossa. There are three terminal branches of the pudendal nerve. The first branch is the inferior rectal (anal) nerve. It exits the nerve just before or inside of Alcock's canal. The other terminal branches are the peroneal nerve and the dorsal nerve of the penis or clitoris.

Pudendal neuropathy is most often a bilateral process. It can be secondary to compression, stretch, direct trauma and radiation. Pudendal neuralgia is a functional entrapment syndrome where pain exacerbation is related to compression or stretch maneuvers. The nerve is compressed when sitting and can be damaged by cycling or motor-cross. The nerve can be stretched during childbirth, and by straining with constipation. Fitness exercise such as weight lifting with squats or leg press, karate, kick boxing and rollerblading have been implicated. Falls onto the buttocks can cause pudendal neuralgia. Iatrogenic causes include vaginal and peri-anal surgery. Radiation neuropathy has been reported following treatment for prostate carcinoma.

Pudendal nerve blocks were initially performed using a transvaginal approach with a finger cot. Obstetricians can manually palpate the ischial spine and direct the needle at it. The ischial spine can be targeted with C-arm fluoroscopy for fluoroscopically directed blockade at the ischial spine. Ultrasound guidance has been described using a transperineal approach and identifying the pudendal artery [9]. This technique requires nerve stimulation to help differentiate the pudendal nerve from the sciatic nerve. CT [4-6] and MRI [10] guidance have been described as means to guide targeting the nerve as it passes between the sacrospinous and sacrotuberous ligaments, at the ischial spine and in Alcock's canal. Targeting the nerve between the sacrospinous and sacrotuberous ligaments puts the patient at risk of inadvertent blockade of the sciatic nerve.

The symptoms can sometimes speak to the level of the entrapment. Symptoms that are primarily related to the peri anal region are often related the impingement of the inferior rectal nerve at the ischial spine or between the sacrospinous and sacrotuberous ligaments. Symptoms that are predominately related to the peroneal or penile (clitoral) nerve branches are likely to be related to impingement in Alcock's canal. MRI evaluation has elucidated four entrapment subcategories. Entrapment can be seen at 1) the level of the piriformis muscle in the sciatic notch; 2) the ischial spine and sacrotuberous ligament; 3) Alcock's canal; and 4) the distal branches. The vast majority of the compression is seen at Alcock's canal.

Pudendal nerve blocks have been used for obstetric analgesia for decades. Pudendal nerve blocks have been used to relieve urethral spasm in paraplegics. Pudendal nerve blocks are a component of the diagnostic algorithm for the diagnosis of pudendal neuropathy. Patients who respond to pudendal nerve blocks are more likely to respond well to pudendal nerve release surgery. Our study demonstrates that interventional radiologist can safely perform CT guided pudendal nerve blocks.

\section{Conclusion}

These results suggest that pudendal nerve block can be performed with CT guidance in a safe and effective manner. Further evaluation of the long-term effectiveness of the procedure with a prospective study is warranted.

\section{REFERENCES}

[1] S. J. Antolak, "Pudendal Neuralgia; Pudendal Nerve Entrapment, Alcock Canal Syndrome, and Pudendal Canal Syndrome,” In: J. M. Potts, Genitourinary Pain and Inflammation: Diagnosis and Management, Humana Press, Totowa, 2008, pp. 39-56.

[2] K. Stav, P. L. Dwyer and L. Roberts, "Prudendal Neuralgia Fact or Fiction?” Obstetrical and Gynecological Survey, Vol. 64, No. 3, 2009, pp. 190-199. doi:10.1097/OGX.0b013e318193324e

[3] J. T. Benson and K. Griffis, "Pudendal Neuralgia, a Severe Pain Syndrome,” American Journal of Obstetrics \& Gynecology, Vol. 192, No. 5, 2005, pp. 1663-1668. doi:10.1016/j.ajog.2005.01.051

[4] D. Thoumas, A. M. Leroi, J. Mauillon, et al., "Pudendal Neu-Ralgia: CT-Guided Nerve Block Technique,” Abdominal Imaging, Vol. 24, No. 3, 1999, pp. 309-312. 


\section{doi:10.1007/s002619900503}

[5] D. M. Hough, K. H. Wittenburg, W. Pawlina, et al., "Chronic Peroneal Pain Caused by Pudendal Nerve Entrapment: Anatomy and CT-Guided Perineural Injection Technique,” American Journal of Roentgenology, Vol. 181, No. 2, 2003, pp. 561-567.

[6] O. Calvillo, I. M. Skaribas and C. Rockett, “Computed Tomography-Guided Pudendal Nerve Block. A New Diagnostic Approach to Long-Term Anoperineal Pain: A Report of Two Cases," Regional Anesthesia and Pain Medicine, Vol. 25, No. 4, 2000, pp. 420-423.

[7] G. Amerenco, Y. Lanoe, M. Perrigo and H. Goudal, “A New Canal Syndrome: Compression of the Pudendal Nerve in Alcock's Canal or Perineural Paralysis of Cyclists,” Pressure Medicine, Vol. 16, No. 8, 1987, p. 399.
[8] J. J. Labat, T. Riant, R. Robert, et al., “Diagnostic Criteria for Pudendal Neuralgia by Pudendal Nerve Entrapment (Nantes Criteria)," Neurourology and Urodynamics, Vol. 27, No. 4, 2008, pp. 306-310. doi:10.1002/nau.20505

[9] P. W. Peng and P. S. Tunber, "Ultrasound Guided Interventional Procedures for Patients with Chronic Pelvic Pain-A Description of Techniques and Review of Literature,” Pain Physician, Vol. 11, No. 2, 2008, pp. 215224.

[10] A. Filler, "Diagnosis and Management of the Pudendal Nerve Entrapment Syndromes: Impact of MR Neurography and Open MR Guided Injections,” Neurosurgery Quarterly, Vol. 18, 2008, pp. 1-6. doi:10.1097/WNQ.0b013e3181642694 